\title{
Defining Rural In Aotearoa New Zealand: A Novel Geographic Classification For Health Purposes
}

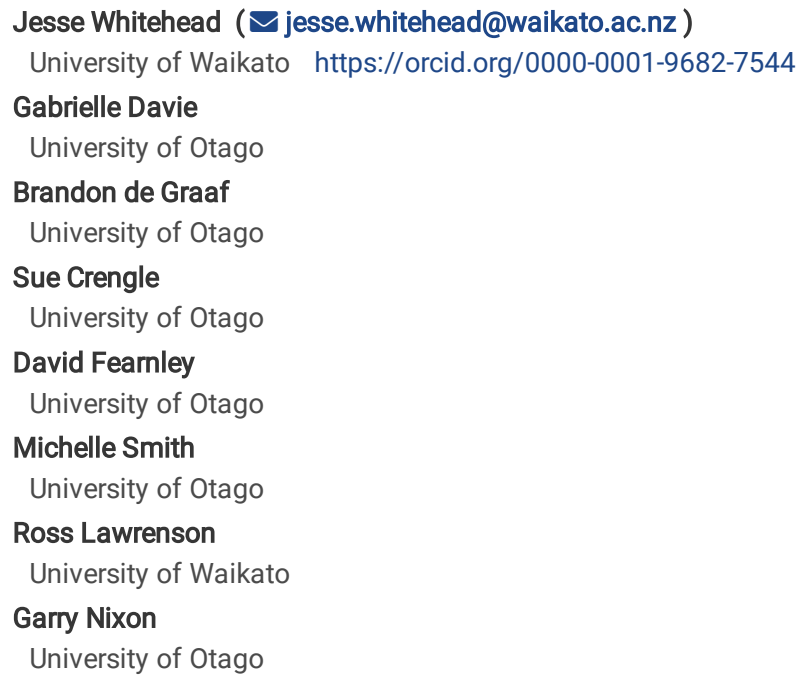




\section{Abstract}

Objectives: To develop a valid rurality classification for health purposes in Aotearoa New Zealand (NZ) that is technically robust and incorporates heuristic understandings of rurality.

Setting: Our Geographic Classification for Health $(\mathrm{GCH})$ is developed for all of NZ.

Participants: We examine the distribution of the entire NZ population across rurality classifications, and use the National Mortality Collection to examine previously masked rural-urban differences in mortality.

Outcome measures: Unadjusted all-cause mortality rates and rural:urban incidence rate ratios (IRRs).

Results: The GCH modifies key population and drive time thresholds in the generic rurality classifications, thereby identifying $19 \%$ of the NZ population as rural. Rural and urban all-cause mortality rates and associated rural:urban IRRs vary considerably depending on rurality classification. The GCH finds a rural mortality rate $21 \%$ higher than for urban areas.

Conclusions: The GCH identifies a distinct rural population, and highlights rural-urban inequities that are masked by generic classifications.

\section{Strengths And Limitations Of This Study}

- This is the first mixed-methods approach to developing a robust rurality classification for health purposes

- The classification has been validated both quantitatively and qualitatively

- Limitations include that the mortality rates calculated are unadjusted and all-cause. Further research in this area using the GCH is planned

\section{Introduction}

\section{Background}

How rurality is defined matters, both from a policy and service delivery perspective, and for the populations and communities who live in rural places. ${ }^{1}$ Definitions of rurality have an important role in health contexts. A fit-for-purpose definition allows the health status of rural populations to be accurately monitored. This may identify rural-urban health inequities, providing the impetus for targeted strategy, policy and interventions for the equitable allocation of resources. ${ }^{2-6}$ There is, however, no internationally agreed definition of 'rural'. Definitions are dependent on context, can change over time, and have become increasingly blurred. ${ }^{1}$ To date, Aotearoa New Zealand (NZ hereafter) has lacked an urban-rural classification designed for use in health research and policy.

\section{Defining rural in geography}

Geographers have long contested the definition of rurality. ${ }^{7}$ The two main ways of conceptualizing and defining rurality are: (1) a socio-cultural approach; and (2) a descriptive, data driven approach. ${ }^{8}$ Socio-cultural definitions consider particular cultural characteristics of communities in order to define places as rural or urban. ${ }^{9}$ Descriptive approaches are technical and quantitative ways to empirically describe socio-spatial characteristics, and classify places as rural if they meet pre-defined criteria. ${ }^{8}$ Both approaches have limitations, particularly when used alone, and can result in anomalies. Socio-cultural approaches assume that population density affects behaviour and attitudes and that values and behaviours differ between rural and urban residents, despite contradictory evidence such as supposedly 'rural' communities in the East End of London. ${ }^{8}$ Conversely, descriptive definitions of rurality have been strongly critiqued as providing an inadequate view of the social construction that is rurality. ${ }^{1,10,11}$ It is also argued that they assume a clear geographic distinction between rural and urban areas, when in fact borders are often blurred, contested, and subjective, ${ }^{11}$ and complex 'black-box' methods reify methodologies and lend results a misleading aura of authenticity. ${ }^{1}$ The core concepts and measures of rurality have not changed markedly since the 1970 s. ${ }^{1}$ Population size and proximity to metropolitan areas are still the most commonly used input variables in rurality measures. However, there is growing recognition that 'rurality' is a fluid concept that is context-dependent. ${ }^{1}$ Rurality is increasingly viewed as a social construct that is defined by discourse. ${ }^{8}$ Social representation is used to consider how people construct themselves as being rural, recognizing that rurality is 'a state of mind' and in the eye of the beholder. ${ }^{8}$ The development of a meaningful classification of rurality needs to effectively balance 'technical' and 'discourse' approaches so that classifications are the result of a clear, transparent, and replicable process, and also make sense on the ground. Heuristic, on the ground understandings of rurality are as important as purely geographic data-driven approaches, and the plurality of rural must be embraced. ${ }^{9}$

The statistician George Box's argues that "all models are wrong, but some are useful". ${ }^{12}$ Descriptive, technical approaches are important, especially in research and policy contexts where quantitative measures are needed to consistently define populations or designate policies. ${ }^{1}$ However, it is somewhat naïve to think that a purely technical approach will produce the most fit-for-purpose classification of rurality - a concept which is multifaceted, nuanced, and dependent on context. $^{1,7-9}$

\section{Defining rural for health}


Internationally, a wide range of approaches to describing areas as urban or rural in a health context have been taken by both researchers and government departments. In the United States of America, there are five key measures of rurality for epidemiological studies, all based on a combination of population size, density, and distance or commuting patterns. ${ }^{13}$ Canada has at least four different rurality classifications used in health research - all based on a combination of population size, density, and distance. ${ }^{14}$ While exact thresholds cannot be universally applied, factors of population size, density, and distance are key considerations in international geographic classifications of rurality. For example, the Modified Monash Model (MMM) was developed in Australia as a framework for distributing rural health workforce recruitment and retention funding. Although six indicators of rural and remote workforce retention were examined, population size and distance to metropolitan centre were found to be more sensitive indicators of the need for recruitment and retention incentives, leading to a more parsimonious classification. ${ }^{6}$

The United States Rural Policy Research Institute ${ }^{15}$ offers guidance on addressing the plurality of rurality when developing a classification for health. It acknowledges that a transparent data-driven geographic approach is preferred to intuition or personal experience. However, it also argues that geographic approaches must be combined with qualitative evaluation and 'ground truthing' to ensure the final classification has face validity. As NZ's Minister of Health, Andrew Little, noted in his keynote address at the 2021 NZ National Rural Health Conference, ${ }^{16}$ the definition of rural is "not just semantic" and has real implications in terms of policy decisions and resource allocation. Poorly defined rural-urban divisions lead to poorly defined and implemented policies. ${ }^{1}$ While descriptive approaches remain an important aspect of classifying rurality to allow meaningful analysis of health data, and thereby inform health policy, ${ }^{1}$ they must involve a qualitative aspect to ensure face validity. ${ }^{9}$

\section{Defining rural in the NZ health context}

The definition of rurality is also an essential component of research that explores rural-urban health inequities. Such inequities, exacerbated by deprivation and ethnicity, have been clearly described internationally. ${ }^{17-19}$ However, the same inequities have not been as clearly demonstrated in NZ. Many health practitioners, academics, and other informed stakeholders argue that this is due to the definitions of 'rural' used. Internationally, it has been demonstrated that different rurality classifications result in inconsistent categorization of areas and populations, impacting the results of epidemiological studies and health service research and thereby potentially masking inequities. ${ }^{20-25}$ This is an example of the Modifiable Area Unit Problem (MAUP), which has shown that the results of analysis can vary according to the size, number and configuration of spatial units that are used. ${ }^{26}$ Furthermore, aggregation methods can greatly influence results, even when the size of the total population is similar between methods. ${ }^{27}$ Similarly, the choice of rurality classification also influences results, as different classifications aggregate together different populations into rural or urban categories. This is problematic in NZ, where a wide range of usually generic definitions of rurality, have been used in the health research literature. ${ }^{28}$

A review of the last 20 years of $\mathrm{NZ}$ health research ${ }^{28}$ revealed the use of over 30 classifications of rurality. This complicates discussions of rural health, and highlights the need for a clear, consistent, and fit-for-purpose definition of rurality in NZ that can be routinely used to accurately monitor rural-urban differences in health outcomes. Statistics New Zealand's (Stats NZ) Urban Rural Experimental Profile (UREP) ${ }^{29}$ is a commonly used rurality classification in the NZ health literature. Although, the National Health Committee in 2010 found little difference in health outcomes between rural and urban residents, ${ }^{30}$ this conclusion is likely an artefact of the way the UREP was used in their analysis. ${ }^{31}$ When the UREP was modified to better represent 'rural' as understood by NZ's rural health community, the relative incidence of heart disease in the 'rural' population increased from $62 \%$ to $166 \%$ of the urban incidence. ${ }^{30-32}$ One issue is that, within an urban-rural binary, the UREP classifies as 'independent urban communities' numerous places that are, in a health discourse, invariably considered rural. Independent urban communities are often rural towns with a considerably smaller population than metropolitan centres. They do not have a significant functional relationship with main urban areas, and tend to have higher levels of socioeconomic deprivation. The UREP category 'Rural areas with high urban influence' is also problematic. These places are, generally, NZ's most affluent, with 'a significant proportion' of residents working in the adjacent 'large urban area'. ${ }^{29}$ In 2018, Stats NZ, updated its Statistical Standard for Geographic Areas (SSGA18) ${ }^{33}$ creating Statistical Area 1s (SA1s) as the smallest geographic unit for census population data. In 2020 Stats NZ's Urban Accessibility (UA) classification ${ }^{34}$ replaced the UREP. The UA was designed to recognize the impact that proximity to urban centres has when determining gradations of rurality. However, the UA remains a 'generic' classification that was not specifically designed for health outcome analyses, and complexities around rural and urban fringes ${ }^{11}$ have not been considered from a health perspective. The UA is therefore likely to continue masking rural-urban health inequities.

There has been a pressing need for a rural-urban classification which supports the consistent analysis of national health data and is likely to have significant uptake and use among health researchers and policymakers. The objective of this paper is to describe the development and validation of a Geographic Classification for Health (GCH) that is not only descriptive and technically robust for use within policy and research contexts, but also aligns with a heuristic sense of what is understood to be rural in the NZ health context.

\section{Methods}

A mixed-methods approach to co-designing and developing a GCH was used. Using this framework all areas of NZ were classified as into one of five urban (U1 or U2) or rural (R1, R2, or R3) categories. While rural-urban classifications for health purposes developed internationally do not directly translate to the NZ context, the criteria that underpin them are highly relevant. To determine appropriate thresholds and develop a fit-for-purpose geographic classification of rurality, key criteria outlined in the international literature were examined. ${ }^{15,35-37}$ The key criteria and details of how these were addressed in the development of the $\mathrm{GCH}$ are described elsewhere ${ }^{38}$ and are available as supplementary material (S1).

\section{Key steps}


The development, testing, and use of the GCH outlined in this paper followed five key steps (Fig. 1). The key co-design partners in developing the GCH were the Ministry of Health's (MoH) National Rural Health Advisory Group (NRHAG), whose members include representatives of the MoH, the New Zealand Rural General Practice Network Rural Health Alliance (RHANZ), Primary Health Organizations (PHOs), District Health Boards (DHBs), the Royal NZ College of General Practitioners (RNZCGP) and rural Māori (the Indigenous population of New Zealand) healthcare providers.

\section{Step 1: Defining the purpose and parameters of the $\mathrm{GCH}$}

The purpose and parameters of the GCH were discussed and finalized among the research team and our co-design partners, NRHAG.

\section{Step 2: Developing a quantitative framework for the $\mathrm{GCH}$}

Next, a transparent quantitative model was developed based on high quality data and clear criteria developed from key rural health documents and research. ${ }^{30,39-41}$ Both the $\mathrm{MoH}$ and Stats NZ indicated that the $\mathrm{GCH}$ would likely be more widely adopted if it was derived from the same building blocks as the UA: SA1s, population size, and drive time data. Using SA1s as the geographic unit ensures compatibility with census-based data, such as ethnicity and area-level socioeconomic deprivation. The SSGA18 Review ${ }^{33}$ and development of the UA ${ }^{34}$ followed international best practice, was detailed and robust, and underwent rigorous testing. Detailed information on the development of the UA is available elsewhere. ${ }^{34}$ The UA classifies each SA1 as belonging to one of eight gradations of urbanicity that can be aggregated to a binary rural-urban variable. The urban core comprises of towns and cities with a population of 10,000 or more, and is broken down into three categories depending on population size: Major, Large or Medium urban areas. All other SA1s are assigned one of five levels of urban accessibility, ranging from "high" to "very remote" depending, on the travel time to the edge of an urban area, as detailed in Table 1.

Table 1

Population and travel time thresholds used in the UA

\begin{tabular}{|c|c|c|c|c|c|}
\hline \multirow[t]{2}{*}{ UR2018 Category } & \multicolumn{5}{|c|}{ The Urban Accessibility Classification } \\
\hline & High Urban Accessibility & Medium Urban Accessibility & Low Urban Accessibility & Remote & Very Remote \\
\hline $\begin{array}{l}\text { Major Urban } \\
\text { (Population } \geq 100,000 \text { ) }\end{array}$ & $0-15 \min$ & $16-25 \mathrm{~min}$ & $26-60 \mathrm{~min}$ & $61-120 \mathrm{~min}$ & $>120 \mathrm{~min}$ \\
\hline $\begin{array}{l}\text { Large Urban } \\
(30,000-99,999)\end{array}$ & & $0-25 \min$ & $26-60 \mathrm{~min}$ & $61-120 \mathrm{~min}$ & $>120 \mathrm{~min}$ \\
\hline $\begin{array}{l}\text { Medium Urban } \\
(10,000-29,999)\end{array}$ & & $0-15 \min$ & $16-60 \mathrm{~min}$ & $61-120 \mathrm{~min}$ & $>120 \mathrm{~min}$ \\
\hline
\end{tabular}

The framework and data behind the GCH are based on population size, density, and drive time as measured in the SSGA18 and UA. Important modifications were made - through co-design workshops with NRHAG and consultation with stakeholders - to the population size and drive time thresholds to better reflect understandings of rurality in health. Particular consideration was paid to the NZ health context, including principles such as the agreement between the MoH and $\mathrm{PHO}$ s for 24 -hour primary care ${ }^{40}$ and the 'Golden Hour.' ${ }^{39}$ Detailed reasoning behind the adjustments to each population and travel time threshold is available elsewhere. ${ }^{42}$

\section{Step 3: Co-design and consultation process}

In addition to working with NRHAG to co-design the GCH, extensive consultation was undertaken with key stakeholders between March 2020 and February 2021. This involved both face-to-face and virtual seminars and workshops with more than 20 organizations and over 300 individuals from a range of sectors. Participants included likely end-users of the $\mathrm{GCH}$ - such as health researchers and policymakers, organizations involved in the delivery of health services such as DHBs and PHOs - as well as representatives of rural communities and health professional groups. Seminars and workshops involved an explanation of the GCH's purpose, the methodology and framework used to develop it, the generic UA, and proposed versions of the GCH (which were modified iteratively throughout the consultation period). Attendees identified their preferred classification and provided feedback on the framework and methodology used to develop the $\mathrm{GCH}$.

\section{Step 4: Testing the validity of the GCH}

In addition to ensuring the $\mathrm{GCH}$ has 'on-the-ground' validity through co-design and consultation, each proposed version was quantitatively tested. The ability of the GCH to accurately classify patients enrolled in urban and rural general practices was compared to the UA and UREP. Although travel for primary healthcare is complex and often involves bypass behaviors, ${ }^{43}$ it can be assumed that most patients living in urban areas enrol with urban GP practices, and most patients living in rural areas likewise enrol with rural clinics. For funding purposes, local Rural Service Alliance Teams (RSLATS, comprised of local community and primary care provider representatives) are responsible for identifying rural practices in their region. Based on criteria laid out in the Rural Ranking Scale $\mathrm{A4}^{45}$ and local knowledge, RSLATs developed a local formula for the allocation rural funding. This process involved considerable consultation and the agreement of more than $75 \%$ of practices was required. Two PHOs that had successfully completed this process, Mahitahi Hauora and WellSouth, provided anonymized patient enrolment data and a list of the urban or rural funding status of all practices in their region. This was used to determine whether patients enrolled in rural practices were living in rural locations. Comparisons were undertaken to estimate how well the UREP, UA, and various versions of the $\mathrm{GCH}$ aligned with PHO enrolment-based rurality. The 'accuracy' of each classification was calculated as the percentage of patients for whom the binary 
urban/rural indicator matched the urban/rural indicator in the PHO enrolment data; $95 \%$ Confidence Intervals (95\%Cls) are provided for each accuracy estimate.

\section{Step 5: Describing rural populations and identifying differences in health outcomes}

The GCH, UA, and UREP classifications were used to describe the 'rural' population of NZ. Detailed examination as to whether rural-urban differences in health outcomes were masked by the use of inappropriate 'generic' classifications of rurality will be presented elsewhere. To provide an indication of the impact of different classifications, in this paper crude mortality rates for urban and rural residents were calculated using the GHC, UA, and UREP. Incidence rate ratios (IRRs) that compare, on a relative scale, the mortality incidence rate for rural residents with that for urban residents are provided with $95 \%$ Confidence Intervals (95\%Cls).

\section{Ethics}

Ethical approval for this research was obtained from the University of Otago Human Research Ethics Committee (reference number HD19/069), and consultation was undertaken with the Ngāi Tahu Research Consultation Committee.

\section{Patient and public involvement statement}

While it was not appropriate or feasible to involve patients and the wider public in the development of research questions, design, and conduct of the study, we have actively engaged the health sector in this process.

\section{Results}

\section{Purpose}

The $\mathrm{GCH}$, a geographic classification with two urban categories $(\mathrm{U} 1, \mathrm{U} 2)$ and three rural categories (R1, R2, R3) was developed with the intent of it being used to accurately monitor rural-urban differences in health outcomes.

\section{Co-design and stakeholder feedback}

When presented with the approach behind the $\mathrm{GCH}$, participants stated that the $\mathrm{GCH}$ methodology was robust. After viewing maps of the GCH and UA, participants indicated that the $\mathrm{GCH}$ was an appropriate classification for their region. Participants often had in-depth local knowledge of their regions, and were able to rapidly determine which of the versions 'made the most sense on the ground'.

\section{Quantitative validation}

Using the PHO enrolment data as the gold standard, the accuracy of the UREP was estimated to be $70.3 \%(95 \% \mathrm{Cl} 70.2 \%, 70.5 \%)$ for WellSouth patients and $65.8 \%(95 \% \mathrm{Cl} 65.5 \%, 66.0 \%)$ for Mahitahi patients. In comparison, the accuracy of the UA was higher (WellSouth: $80.8 \%$ (95\%Cl 80.6\%, 80.9\%); Mahitahi: $81.3 \%,(95 \% \mathrm{Cl} 81.1 \%, 81.4 \%)$ ), while the accuracy of the $\mathrm{GCH}$ was higher still (WellSouth: $94.7 \%,(95 \% \mathrm{Cl} 94.6 \%, 94.8 \%)$; Mahitahi: $92.5 \%,(95 \% \mathrm{Cl} 92.3 \%$, $92.6 \%))$.

\section{The Geographic Classification for Health}

The final population and drive-time thresholds used in the $\mathrm{GCH}$ are outlined below in Table 2. Details of border issues and additional considerations are provided in supplementary material (S2). Figure 2 and Fig. 3 show the GCH for the North and South Islands of NZ respectively.

Table 2: Population and travel time thresholds used in the $\mathrm{GCH}$

\begin{tabular}{|c|c|c|c|c|c|}
\hline \multirow[b]{3}{*}{ UR2018 Category } & \multicolumn{5}{|c|}{ Geographic Classification for Health } \\
\hline & \multicolumn{2}{|l|}{ Urban } & \multicolumn{3}{|l|}{ Rural } \\
\hline & $\begin{array}{l}\text { Urban } 1 \\
\text { (U1) }\end{array}$ & $\begin{array}{l}\text { Urban } 2 \\
\text { (U2) }\end{array}$ & $\begin{array}{l}\text { Rural } 1 \\
\text { (R1) }\end{array}$ & \begin{tabular}{|l} 
Rural 2 \\
(R2)
\end{tabular} & $\begin{array}{l}\text { Rural } 3 \\
\text { (R3) }\end{array}$ \\
\hline $\begin{array}{l}\text { Major urban } \\
\text { (Population } \geq 100,000 \text { ) }\end{array}$ & $\leq 25 \mathrm{~min}$ & & $\begin{array}{l}>25 \text { to } \leq 60 \\
\min \end{array}$ & $\begin{array}{l}>60 \text { to } \leq 90 \\
\min \end{array}$ & $>90 \mathrm{~min}$ \\
\hline $\begin{array}{l}\text { Large Urban } \\
(30,000-99,999)\end{array}$ & & $\leq 20 \mathrm{~min}$ & $\begin{array}{l}>20 \text { to } \leq 50 \\
\min \end{array}$ & $\begin{array}{l}>50 \text { to } \leq 80 \\
\min \end{array}$ & $>80 \mathrm{~min}$ \\
\hline $\begin{array}{l}\text { Medium Urban } \\
(10,000-29,999)\end{array}$ & & & $\leq 25 \mathrm{~min}$ & $\begin{array}{l}>25 \text { to } \leq 60 \\
\min \end{array}$ & $>60 \mathrm{~min}$ \\
\hline $\begin{array}{l}\text { Small Urban } \\
(1,000-9,999)\end{array}$ & & & & $\leq 25 \mathrm{~min}$ & $>25 \mathrm{~min}$ \\
\hline
\end{tabular}

\section{Describing rural populations}

Table 3 displays the New Zealand usually resident Census 2018 population classified us rural or urban under the GCH, the generic UA classification, and the retired UREP. The distribution of selected demographic variables, including age, sex, and ethnicity is also displayed. Figure 4 highlights the overlap between how these populations are classified by each rurality classification. 
Table 3

The population of Aotearoa defined as rural or urban according to the $\mathrm{GCH}$ and two generic rurality classifications

\begin{tabular}{|c|c|c|c|c|c|c|c|c|c|c|c|c|}
\hline \multirow{2}{*}{$\begin{array}{l}\text { Population } \\
\text { variable }\end{array}$} & \multicolumn{3}{|l|}{$\mathrm{GCH}$} & \multicolumn{3}{|c|}{ UA Classification } & \multicolumn{3}{|l|}{ UREP } & \multirow{2}{*}{$\begin{array}{l}\text { Rural in all } 3 \\
\text { classifications }\end{array}$} & \multirow{2}{*}{$\begin{array}{l}\text { Urban in all } 3 \\
\text { classifications } \\
\% \text { Urban }\end{array}$} & \multirow{2}{*}{$\begin{array}{l}\text { Clas } \\
\text { as b } \\
\text { Urbe } \\
\text { and } \\
\%\end{array}$} \\
\hline & Rural & Urban & $\begin{array}{l}\% \\
\text { Rural }\end{array}$ & Rural & Urban & $\begin{array}{l}\% \\
\text { Rural }\end{array}$ & Rural & Urban & $\begin{array}{l}\% \\
\text { Rural }\end{array}$ & & & \\
\hline Total & 892,881 & $3,806,307$ & $19 \%$ & $1,228,680$ & $3,470,508$ & $26 \%$ & 685,209 & $4,013,979$ & $15 \%$ & $9 \%$ & $70 \%$ & $20 \%$ \\
\hline \multicolumn{13}{|l|}{$\begin{array}{l}\text { Age } \\
\text { (years) }\end{array}$} \\
\hline $0-14$ & 175,215 & 747,576 & $19 \%$ & 247,860 & 674,931 & $27 \%$ & 140,616 & 782,175 & $15 \%$ & $10 \%$ & $70 \%$ & $20 \%$ \\
\hline $15-29$ & 142,677 & 820,242 & $15 \%$ & 195,426 & 767,493 & $20 \%$ & 106,086 & 856,833 & $11 \%$ & $7 \%$ & $77 \%$ & $16 \%$ \\
\hline $30-44$ & 148,563 & 755,187 & $16 \%$ & 206,853 & 696,897 & $23 \%$ & 118,224 & 785,526 & $13 \%$ & $8 \%$ & $74 \%$ & $18 \%$ \\
\hline $45-59$ & 190,878 & 741,750 & $20 \%$ & 277,533 & 655,095 & $30 \%$ & 163,401 & 769,227 & $18 \%$ & $11 \%$ & $67 \%$ & $22 \%$ \\
\hline $60-74$ & 166,512 & 506,610 & $25 \%$ & 222,399 & 450,723 & $33 \%$ & 123,171 & 549,951 & $18 \%$ & $12 \%$ & $63 \%$ & $25 \%$ \\
\hline $75+$ & 68,232 & 234,015 & $23 \%$ & 77,802 & 224,445 & $26 \%$ & 33,261 & 268,986 & $11 \%$ & $8 \%$ & $69 \%$ & $24 \%$ \\
\hline \multicolumn{13}{|l|}{ Sex } \\
\hline Male & 447,780 & $1,871,190$ & $19 \%$ & 619,812 & $1,699,158$ & $27 \%$ & 350,964 & $1,968,006$ & $15 \%$ & $10 \%$ & $70 \%$ & $20 \%$ \\
\hline Female & 445,089 & $1,934,784$ & $19 \%$ & 608,871 & $1,771,002$ & $26 \%$ & 334,368 & $2,045,505$ & $14 \%$ & $9 \%$ & $71 \%$ & $20 \%$ \\
\hline \multicolumn{13}{|l|}{ Ethnicity } \\
\hline European & 730,947 & $2,566,236$ & $22 \%$ & $1,025,346$ & $2,271,837$ & $31 \%$ & 587,880 & $2,709,303$ & $18 \%$ & $11 \%$ & $65 \%$ & $24 \%$ \\
\hline Māori & 196,530 & 579,096 & $25 \%$ & 253,461 & 522,165 & $33 \%$ & 125,349 & 650,277 & $16 \%$ & $12 \%$ & $64 \%$ & $25 \%$ \\
\hline Pacific & 27,840 & 353,778 & $7 \%$ & 31,929 & 349,689 & $8 \%$ & 14,163 & 367,455 & $4 \%$ & $2 \%$ & $89 \%$ & $8 \%$ \\
\hline Asian & 36,273 & 671,337 & $5 \%$ & 48,984 & 658,626 & $7 \%$ & 23,634 & 683,976 & $3 \%$ & $2 \%$ & $92 \%$ & $6 \%$ \\
\hline MELAA & 6,765 & 63,867 & $10 \%$ & 7,188 & 63,444 & $10 \%$ & 3,963 & 66,669 & $6 \%$ & $4 \%$ & $87 \%$ & $10 \%$ \\
\hline Other & 11,700 & 46,251 & $20 \%$ & 16,566 & 41,385 & $29 \%$ & 9,996 & 47,955 & $17 \%$ & $11 \%$ & $68 \%$ & $22 \%$ \\
\hline
\end{tabular}

\section{Differences in health outcomes}

For NZ, rural and urban all-cause mortality rates and associated rural:urban incidence rate ratios (IRRs) vary considerably depending on the classification used (Table 4). Using UREP, the IRR estimates the mortality rate for rural residents at $67 \%$ that of urban residents whereas the $\mathrm{GCH}$ estimates the rural mortality rate as $21 \%$ higher than for urban.

Table 4

Crude all-cause mortality rate per 100,000 person-years for rural and urban areas of NZ

\begin{tabular}{|lllll|}
\hline Classification & \multicolumn{2}{l}{ Mortality } & Rate* & \multicolumn{2}{l|}{ Incidence Rate Ratio } \\
\hline & Rural & Urban & Estimated & $95 \% \mathrm{Cl}$ \\
\hline UREP & 498 & 743 & 0.67 & $(0.66,0.68)$ \\
UAC & 668 & 722 & 0.93 & $(0.92,0.93)$ \\
\hline GCH & 823 & 681 & 1.21 & $(1.20,1.22)$ \\
\hline *Crude all-cause mortality rate per 100,000 person-years $(2013-2017)$
\end{tabular}

\section{Discussion}

The GCH is a novel rurality classification which delineates rural and urban areas of NZ for health research purposes. It evolved from extensive qualitative and quantitative development and testing and as such 'makes sense on the ground' while being technically robust for use within policy and research contexts. It meets the key criteria for developing rurality classifications that have been described in the literature: ${ }^{15,35-37}$ it is transparent and data-driven; based on parsimonious variables; derived from a replicable process; and is based on a spatial unit that allows for comprehensive coverage, aggregation, and linking to 
key population datasets. Importantly, the GCH aligns with a both heuristic sense of what is rural in a health context, and understandings of rurality as evidenced by primary care enrolments.

The population that the $\mathrm{GCH}$ describes as rural is substantially different from that described by previous classifications. Overall, a higher proportion of people are classified as rural under the GCH compared to the UREP. This is likely due to the inclusion in the GCH of 'Independent urban communities' as rural towns. The UA classification considers an even larger proportion (26\%) of people live in rural areas. However, this is likely to be an artefact driven by the 'peri-urban zone' (areas on the fringes of cities that have high or medium accessibility to urban areas) that is classed as rural in the UA taxonomy. Interestingly, despite the proportion of people classified as rural varying from $15-26 \%$, less than one-tenth of the population was classified as rural under all three of the classifications examined. Around 440,000 people are classified as rural regardless of whether the GCH, UA, or UREP is used. However, there are roughly 500,000 people who would be considered rural under the UA and/or UREP that are classed as urban by the GCH. A further 148,000 people who are urban under both the UA and UREP are recognized as rural by the $\mathrm{GCH}$. This lack of overlap between the populations described by the three classifications partially reflects the different methodologies underpinning them. While all classifications use population size and density, the UREP categories are based on Census commuting data, while the UA is derived from drive-time to metropolitan areas. The GCH extends this through a mixed-methods approach to determining appropriate thresholds. It is important that the $\mathrm{GCH}$ describes a substantially different rural population as understood within a health context. The three different populations described by the GCH, UA, and UREP are also likely to have different health characteristics, as evidenced by higher rural mortality rates when the $\mathrm{GCH}$ is used to define rurality. These initial findings indicate that previous classifications may have masked rural-urban differences in health outcomes. The fit-for-purpose definition of rurality provided by the $\mathrm{GCH}$ may contribute to unmasking other rural-urban variations in health outcome.

The classification of rurality used when undertaking health research or designing health policy is therefore very important, and can influence results somewhat akin to the MAUP. ${ }^{26}$ Uncritically selecting a classification, which may not have been designed to address the research question, could produce misleading results. Researchers and policy makers both in NZ and internationally will need to understand the details and concepts behind rurality classifications used in previous health research when drawing conclusions and developing policy. Transparency in the development, selection, and use of rurality classifications is essential to ensure that the results of health research can be meaningfully compared over time.

While the GCH classification is specific to NZ, its development is of international relevance. Our approach embraces 'the two-ness of rural' while classifying rurality. ${ }^{9,15}$ This shows that mixed-methods can be used to design, develop, and test a technically robust and heuristically valid rurality classification that is not only useful in policy and research settings, but also reflects on-the-ground understandings of rurality. To our knowledge, this paper is the first to describe a mixed-methods approach to developing a technically robust and qualitatively valid geographic classification of rurality for health research and policy purposes.

Inconsistent definitions of rurality in NZ health research have hindered understandings of rural health outcomes, subsequently limiting the development of specific rural health policies and interventions. Different rurality classifications identify different 'rural' populations and this has important implications for health policy and funding. These populations will have distinct health needs and require different services. While the GCH can describe a population as rural, it has not been designed to uncritically guide health policy and funding decisions. It is not a formula for distributing health resources or funding, nor is it an index of healthcare accessibility or workforce shortage. Users must be aware of the limitations of a purely geographic classification of rurality. Additional data and local knowledge are crucial when making policy or funding decisions. This could include: the distribution of population sub-groups; the locations of health services and workforce shortage; and the distribution of the social determinants of health. ${ }^{46}$ However, we foresee the GCH as being a useful tool in health outcome analysis and hope that the results of future research will guide the development of comprehensive, evidence-based rural health policy in NZ. The development of the GCH is the first component of a wider Health Research Council of New Zealand funded project, the second phase of which will extend this work by analysing a range of health outcomes by rurality identifying whether rural-urban differences have previously been masked by generic classifications. ${ }^{38}$

\section{Conclusion}

This paper outlines a novel approach to developing a rurality classification for health that embraces both the technical and heuristic aspects of rurality. Fostering close relationships with co-design partners and ensuring early engagement with stakeholders and end-users are key to ensuring that rurality classifications are fit-for-purpose, valid and likely to be widely used.

\section{Declarations}

\section{Contributorship Statement}

All authors contributed to the conception, design and interpretation of this work, as well as editing the manuscript and providing final approval for publication. JW led the technical development of the GCH and drafted the first manuscript, while GD and BdG were responsible for the population and mortality analyses.

\section{Competing interests}

The authors have no competing interests.

\section{Funding}




\section{Data sharing statement}

The GCH will be made publicly accessible, and is available on request. However, for privacy and confidentiality reasons we are unable to make the mortality data used in our analysis publicly available.

\section{Ethics approval and consent to participate:}

Ethical approval for this research was obtained from the University of Otago Human Research Ethics Committee (reference number HD19/069), and consultation was undertaken with the Ngāi Tahu Research Consultation Committee.

\section{Consent for publication:}

Not applicable

\section{Acknowledgements:}

We consulted extensively on the GCH throughout 2020 and would like to thank all the stakeholders for their feedback and comments which have helped to refine and improve the $\mathrm{GCH}$. We would also like to thank our co-design partners NRHAG, as well as our international, domestic, and Māori advisory groups who provided invaluable guidance throughout the development and testing of the $\mathrm{GCH}$. We are also grateful to the geospatial team at Statistics New Zealand who made the data underlying the Urban Accessibility classifiation available to us, and readily engaged in discussions about approaches to classifying rurality. Finally, we would like to acknowledge that this project would not have been possible without the funding of the Health Research Council of New Zealand.

\section{References}

1. Nelson KS, Nguyen TD, Brownstein NA, et al. Definitions, measures, and uses of rurality: A systematic review of the empirical and quantitative literature. Journal of Rural Studies. 2021;82:351-365.

2. Smith KB, Humphreys JS, Wilson MGA. Addressing the health disadvantage of rural populations: How does epidemiological evidence inform rural health policies and research? Australian Journal of Rural Health. 2008;16(2):56-66.

3. Barnidge EK, Radvanyi C, Duggan K, et al. Understanding and Addressing Barriers to Implementation of Environmental and Policy Interventions to Support Physical Activity and Healthy Eating in Rural Communities. The Journal of Rural Health. 2013;29(1):97-105.

4. Attanasio O, Gomez LC, Rojas AG, Vera-Hernández M. Child health in rural Colombia: determinants and policy interventions. Economics \& Human Biology. 2004/12/01/ 2004;2(3):411-438.

5. Grobler L, Marais BJ, Mabunda S. Interventions for increasing the proportion of health professionals practising in rural and other underserved areas. Cochrane Database of Systematic Reviews. 2015(6).

6. Humphreys J, Wakerman J. Learning from history: How research evidence can inform policies to improve rural and remote medical workforce distribution. Australian Journal of Rural Health. 2018;26(5):329-334.

7. Halfacree KH. Locality and social representation: space, discourse and alternative definitions of the rural. Journal of rural studies. 1993;9(1):23-37.

8. Woods M Defining the rural. Rural geography: Processes, responses and experiences in rural restructuring. London: SAGE Publications; $2011: 3-16$.

9. Bell MM. The two-ness of rural life and the ends of rural scholarship. Journal of rural studies. 2007;23(4):402-415.

10. Cloke P (En) culturing political economy: a life in the day of a 'rural geographer'. Writing the rural: Five cultural geographies. 1994:149-190.

11. Woods M Rural geography: blurring boundaries and making connections. Progress in human geography. 2009;33(6):849-858.

12. Box GE. All models are wrong, but some are useful. Robustness in Statistics. 1979;202(1979):549.

13. Hall SA, Kaufman JS, Ricketts TC. Defining urban and rural areas in US epidemiologic studies. Journal of urban health. 2006;83(2):162-175.

14. Muula A How do we define'rurality'in the teaching on medical demography? Rural \& Remote Health. 2007;7(1).

15. Mueller KJ, Coburn AF, Knudson A, et al. Considerations for defining rural places in health policies and programs: Rural Policy Research Institute; 2020.

16. Little A Opening address. Paper presented at: National Rural Health Conference, 2021; Taupō, New Zealand.

17. Australian Institute of Health and Welfare. Rural and Remote Health. https://www.aihw.gov.au/getmedia/6d6c9331-5abf-49ca-827b-e1df177ab0d3/ah165-11-rural-remote-health.pdf.aspx.

18. Eberhardt MS, Pamuk ER. The importance of place of residence: examining health in rural and nonrural areas. American journal of public health. 2004;94(10):1682-1686.

19. Romanow R Rural and remote communities. In: Commission on the Future of Health Care in Canada, ed. Building on values: the future of health care in Canada - Final report, 2002:159-169.

20. Hawley ST, Chang S, Risser D, Zhang Q. Colorectal cancer incidence and mortality in Texas 1990-1992: a comparison of rural classifications. The Journal of rural health. 2002;18(4):536-545. 
21. Inagami S, Gao S, Karimi H, Shendge MM, Probst JC, Stone RA. Adapting the Index of Relative Rurality (IRR) to estimate rurality at the ZIP code level: A rural classification system in health services research. The Journal of Rural Health. 2016;32(2):219-227.

22. Langlois PH, Jandle L, Scheuerle A, Horel SA, Carozza SE. Occurrence of conotruncal heart birth defects in Texas: a comparison of urban/rural classifications. The Journal of Rural Health. 2010;26(2):164-174.

23. West AN, Lee RE, Shambaugh-Miller MD, et al. Defining "rural" for veterans' health care planning. The Journal of Rural Health. 2010;26(4):301-309.

24. Weissman S, Duffus WA, Vyavaharkar M, et al. Defining the rural HIV epidemic: correlations of 3 definitions-South Carolina, 2005-2011. The Journal of Rural Health. 2014;30(3):275-283.

25. Berke EM, West AN, Wallace AE, Weeks WB. Practical and Policy Implications of Using Different Rural-Urban Classification Systems: A Case Study of Inpatient Service Utilization Among Veterans Administration Users. The Journal of Rural Health. 2009;25(3):259-266.

26. Openshaw S, Taylor PJ. A million or so correlation coefficients: Three experiments on the modifiable areal unit problem. In: Wrigley N, ed. Statistical applications in spatial sciences. London: Pion; 1979:127-144.

27. Schuurman N, Bell N, Dunn JR, Oliver L. Deprivation indices, population health and geography: an evaluation of the spatial effectiveness of indices at multiple scales. Journal of urban health. 2007;84(4):591-603.

28. Farrell N, Fearnley D. Spoiled by choices: A scoping review of 20 years of inconsistent rural health definitions Paper presented at: National Rural Health Conference; April 29 - May 1, 2021; Taupō, New Zealand.

29. Statistics New Zealand. An Urban/Rural Profile (experimental). http://archive.stats.govt.nz/browse_for_stats/Maps_and_geography/Geographicareas/urban-rural-profile.aspx.

30. National Health Committee. Rural Health: Challenges of distance, opportunities for innovation. Wellington 2010.

31. Fearnley D, Lawrenson R, Nixon G. 'Poorly defined': unknown unknowns in New Zealand Rural Health. The New Zealand Medical Journal (Online). 2016;129(1439):77.

32. Ministry of Health. Urban-Rural Health Comparisons: Key results of the 2002/03 New Zealand Health Survey. Wellington 2007.

33. Statistics New Zealand. Statistical standard for geographic areas http://archive.stats.govt.nz/methods/classifications-and-standards/classificationrelated-stats-standards/geographic-areas.aspx\#gsc.tab=0.

34. Statistics New Zealand. Urban accessibility - methodology and classification. https://www.stats.govt.nz/methods/urban-accessibility-methodology-andclassification.

35. Hart LG, Larson EH, Lishner DM. Rural definitions for health policy and research. American journal of public health. 2005;95(7):1149-1155.

36. Humphreys J Delimiting 'rural': implications of an agreed 'rurality'index for healthcare planning and resource allocation. Australian Journal of Rural Health. 1998;6(4):212-216.

37. McGrail MR, Humphreys JS. Geographical classifications to guide rural health policy in Australia. Australia and New Zealand health policy. 2009;6(1):28.

38. Nixon G, Davie G, Whitehead J, et al. Developing the Geographic Classification for Health: A research protocol. Australian Journal of Rural Health. in submission.

39. Lilley R, de Graaf B, Kool B, et al. Geographical and population disparities in timely access to prehospital and advanced level emergency care in New Zealand: a cross-sectional study. BMJ open. 2019;9(7):e026026.

40. Ministry of Health. PHO Services Agreement. Version 6. 1 Wellington 2018.

41. Fraser J. Rural health: A literature review for the National Health Committee.

https://www.moh.govt.nz/notebook/nbbooks.nsf/0/A29CD67B3EB1CD03CC2579A5006ECDC7/\$file/rural-health-literature-review.pdf.

42. Whitehead J, Davie G, Graaf Bd, et al. The Geographic Classification for Health: Methodology and classification report, May 20212021.

43. Whitehead J, Pearson AL, Lawrenson R, Atatoa-Carr P. Spatial equity and realised access to healthcare-a geospatial analysis of general practitioner enrolments in Waikato, New Zealand. Rural \& Remote Health. 2019;19(4).

44. Janes R Defining'rural', who decides. New Zealand Doctor. 2006;12(6).

45. London M. The future of rural general practice in New Zealand. The New Zealand Medical Journal (Online). 2004;117(1191).

46. University of Otago. Socioeconomic Deprivation Indexes: NZDep and NZiDep, Department of Public Health.

https://www.otago.ac.nz/wellington/departments/publichealth/research/hirp/otago020194.htm.

\section{Figures}




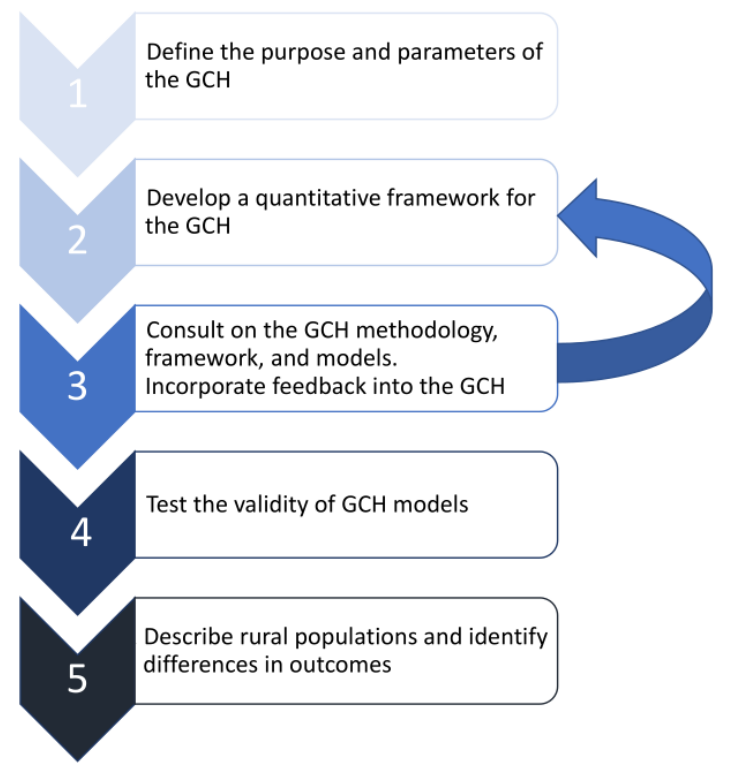

\section{Figure 1}

details the key steps in the development, testing and use of the $\mathrm{GCH}$. 


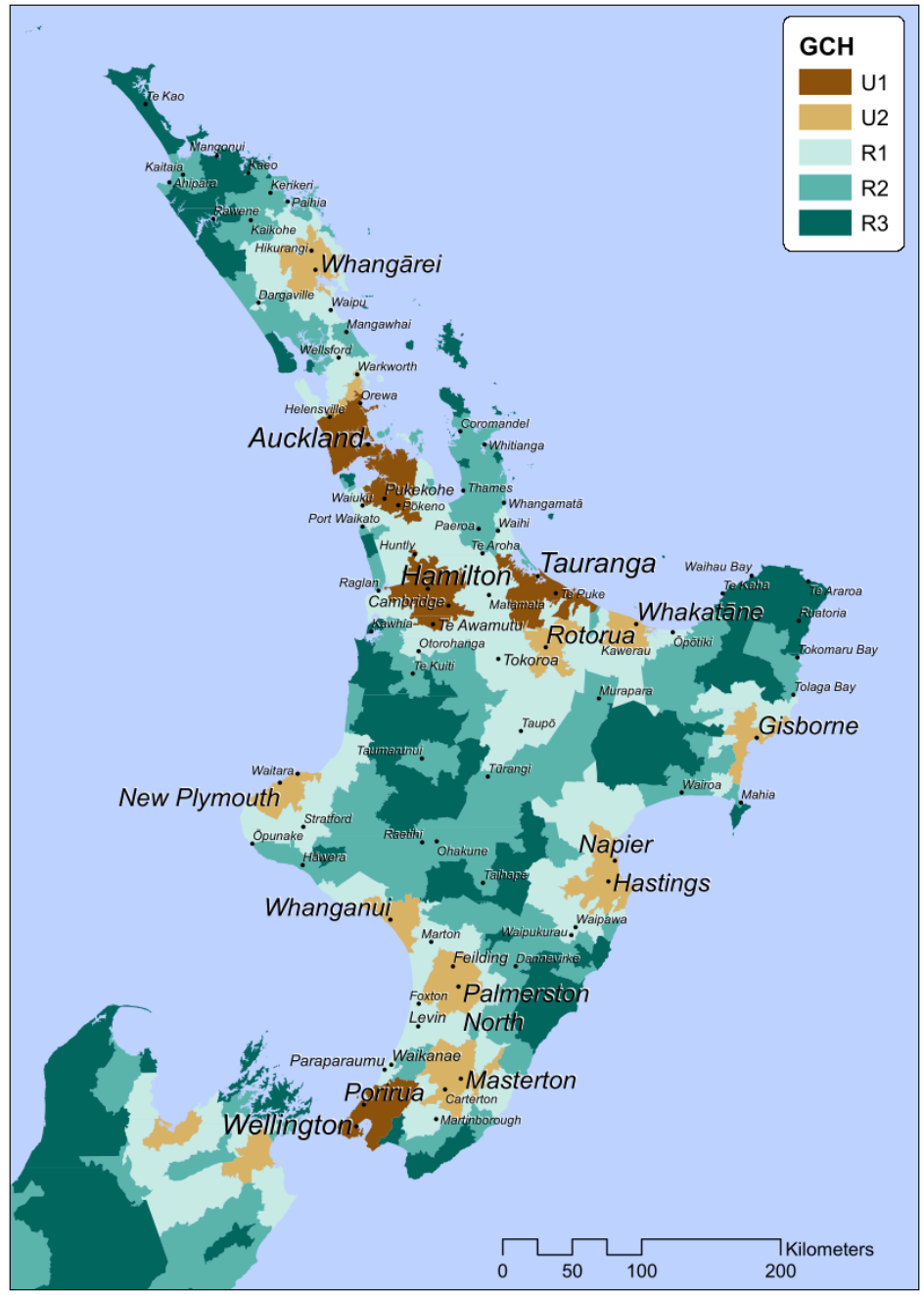

\section{Figure 2}

show the distribution of $\mathrm{GCH}$ categories across the North and South islands of NZ respectively. As noted in the map legend, green represents rural areas while orange/brown represents urban areas. 


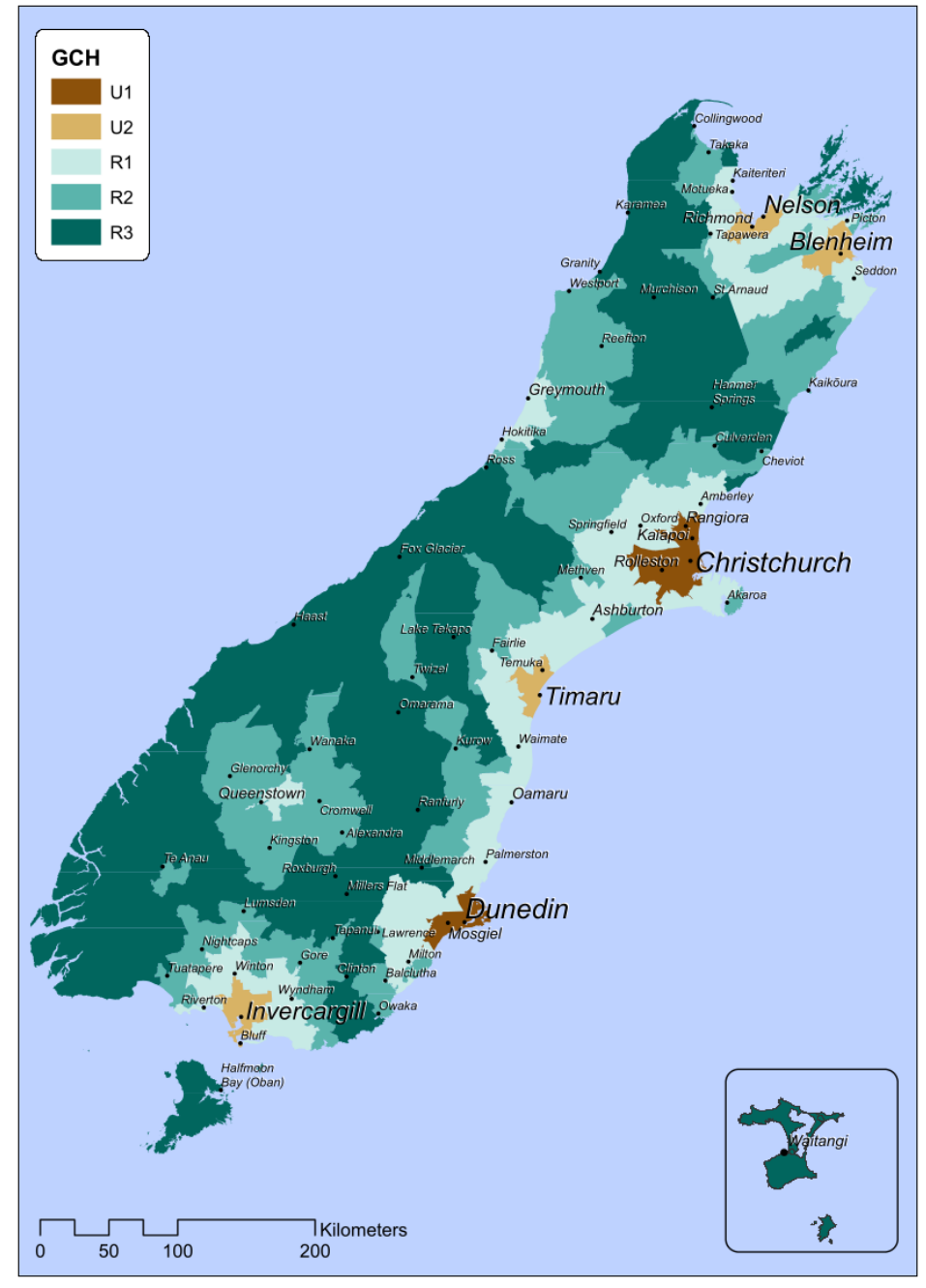

\section{Figure 3}

show the distribution of GCH categories across the North and South islands of NZ respectively. As noted in the map legend, green represents rural areas while orange/brown represents urban areas. 


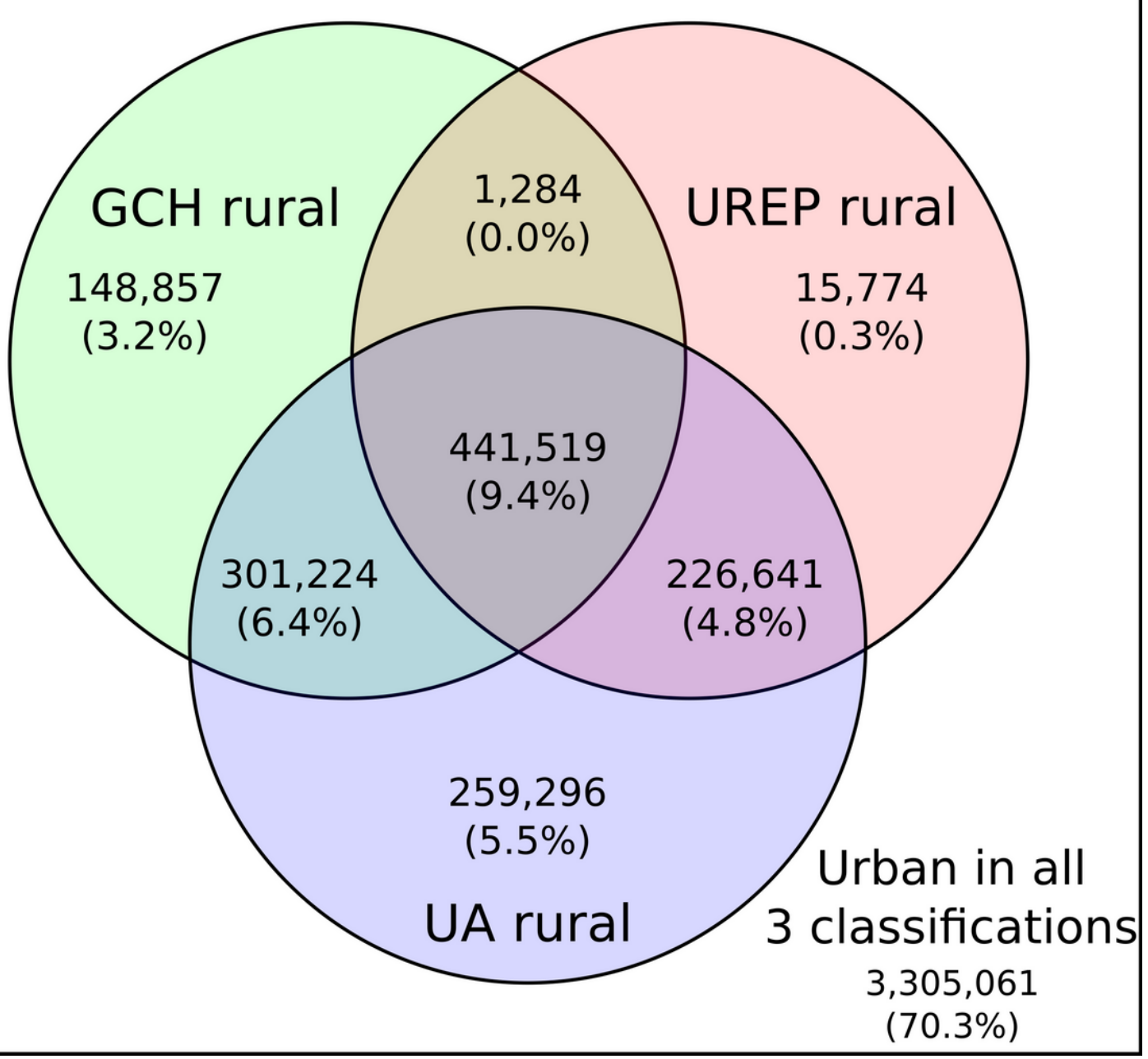

Figure 4

is a Venn diagram representing the populations identified as rural according to three classifications. Overlapping areas reflect populations identified as rural by more than one classification (such as the central area showing that 441,519 people are considered rural by all three classifications). 